\title{
Celastrol alleviates angiotensin II-mediated vascular smooth muscle cell senescence via induction of autophagy
}

\author{
XIAN-JIE XU ${ }^{1,2}$, WEI-BO ZHAO ${ }^{1}$, SHI-BIN FENG ${ }^{1}$, CHENG SUN $^{1}$, QIANG CHEN ${ }^{1}$, BING NI ${ }^{2}$ and HOU-YUAN HU ${ }^{1}$ \\ ${ }^{1}$ Department of Cardiology, Southwest Hospital; ${ }^{2}$ Department of Pathophysiology and High \\ Altitude Pathology, Third Military Medical University, Chongqing 400038, P.R. China
}

Received January 6, 2017; Accepted July 28, 2017

DOI: $10.3892 / \mathrm{mmr} .2017 .7533$

\begin{abstract}
Reactive oxygen species (ROS) production has been implicated in the promotion of cellular senescence. Celastrol, a quinone methide triterpenoid isolated from the Celastraceae family, exerts antioxidant effects and enhances autophagy in various cell types. Since autophagy serves an important role in regulating ROS, it was hypothesized that the antioxidant effect of celastrol is via enhanced autophagy, thus inhibiting cell senescence. Therefore, the present study used a Senescence $\beta$-Galactosidase Staining kit, western blot analysis and cell cycle analysis to investigate whether celastrol alleviates angiotensin (Ang) II-induced cellular senescence by upregulating autophagy in vascular smooth muscle cells (VSMCs). The results demonstrated that celastrol reduced Ang II-induced senescence of VSMCs. Ang II-induced generation of ROS and the subsequent VSMC senescence were counteracted by pretreatment with celastrol, determined by a ROS assay kit. Celastrol significantly upregulated VSMC autophagy, which reduced intracellular ROS and the subsequent cellular senescence induced by Ang II. Furthermore, celastrol markedly suppressed activity of the mechanistic target of rapamycin signaling pathway in VSMCs. In conclusion, the present study demonstrated that celastrol counteracts VSMC senescence probably by reducing ROS production via activation of
\end{abstract}

Correspondence to: Dr Hou-Yuan Hu, Department of Cardiology, Southwest Hospital, Third Military Medical University, 29 Gaotanyan Street, Chongqing 400038, P.R. China

E-mail: houyuanhu@hotmail.com

Dr Bing Ni, Department of Pathophysiology and High Altitude Pathology, Third Military Medical University, 30 Gaotanyan Street, Chongqing 400038, P.R. China

E-mail: nibingxi@126.com

Abbreviations: CeT, celastrol; SA $\beta$-gal, senescence-associated $\beta$-galactosidase; NAC, N-acetyl-L-cysteine; 3Ma, 3-methyladenine; Baf A1, bafilomycin A1; LC3, microtubule-associated protein light chain 3

Key words: celastrol, angiotensin II, reactive oxygen species, senescence, autophagy autophagy, which may hold promise for the prevention and treatment of aging-associated cardiovascular disorders such as atherosclerosis.

\section{Introduction}

The senescence of vascular smooth muscle cells (VSMCs) serves a causal role in the pathogenesis and development of vascular aging, that is closely associated with cardiovascular diseases (1). Senescent cells exhibit increased oxidative stress, telomere dysfunction and stable growth arrest (2) as well as increased expression of senescence-associated $\beta$-galactosidase (SA- $\beta$-gal), p53, p21 and p16 $(3,4)$. High intracellular levels of reactive oxygen species (ROS) are known to induce premature senescence (5). Recent studies suggested that ROS-induced senescence involves reinforcement of positive feedback signaling, further amplifying senescent signals, and ultimately the senescent phenotype $(5,6)$.

Ang II serves an important role in the pathogenesis of various human diseases including atherosclerosis, and inhibition of Ang II activity has been demonstrated to reduce the morbidity and mortality due to cardiovascular diseases (7). Ang II increases ROS production and reduces cellular antioxidant capacity, leading to vascular senescence through a p53/p21-dependent mechanism in VSMCs (4). A growing body of evidence has demonstrated that treatment with biological substances and pharmaceuticals can reduce premature cellular senescence, providing a potential treatment for aging-associated diseases $(8,9)$.

Celastrol is a quinone methide triterpenoid isolated from the traditional Chinese medicinal plant Tripterygium wilfordii Hook f. Celastrol has attracted increasing attention due to its potential therapeutic effects on inflammation and metabolic disorders $(10,11)$. There is accumulating evidence that celastrol can also favorably affect cardiovascular function by preventing circulatory failure (12), atherosclerosis (13) or myocardial ischemic injury (14). Our previous study demonstrated that celastrol exerted inhibitory effects on platelet activation which could contribute to both thrombotic and inflammatory events (15). A recent study reported that a short-term treatment using tripterine (celastrol) reduced ox-low-density lipoprotein (LDL)-induced ROS generation in endothelial progenitor cells (16). It remains unknown whether celastrol counteracts cellular senescence by reducing ROS. 
Among the mechanisms regulating ROS, autophagy serves an important role (17). Autophagy is a type of evolutionarily conserved mechanism that is linked to several cellular signaling pathways and can impact VSMC survival and function. Upregulation of autophagy with stimuli, including intracellular or extracellular factors, can protect VSMCs against cell death $(18,19)$. A recent study reported that defective autophagy in VSMCs accelerates senescence and promotes ligation-induced neointima formation and diet-induced atherogenesis, implying that stimulating autophagy could be a novel strategy in the treatment of arterial diseases (20). In the present study, it was hypothesized that celastrol serves an anti-senescence role by inducing autophagy to reduce ROS production, using VSMCs.

\section{Materials and methods}

Chemicals, reagents and antibodies. Celastrol, N-acetyl-Lcysteine (NAC), 3-methyladenine (3Ma), bafilomycin A1 (Baf A1), rapamycin (Rapa) and dimethyl sulfoxide (DMSO) were provided by Sigma-Aldrich (Merck KGaA, Darmstadt, Germany). Angiotensin II (Ang II) was obtained from EMD Millipore (Billerica, MA, USA). A Senescence $\beta$-galactosidase staining kit (cat. no. 9860), polyclonal rabbit antibodies against LC3A/B (cat. no. 4108), phosphorylated (p)-protein kinase B (Akt; cat. no. 9271), Akt (cat. no. 9272), p-mechanistic target of rapamycin (mTOR; cat. no. 2971), mTOR (cat. no. 2972), p-ribosomal protein S6 kinase $\beta 1$ (p70S6K; cat. no. 9205), p70S6K (cat. no. 9202) and $\beta$-Actin (cat. no. 4967), and the monoclonal rabbit antibody against GAPDH (cat. no. 2118) were all purchased from Cell Signaling Technology, Inc. (Danvers, MA, USA). Monoclonal mouse antibodies against p21 (cat. no. sc-6246) and p53 (cat. no. sc-99) were from Santa Cruz Biotechnology, Inc. (Dallas, TX, USA). Polyclonal rabbit antibodies against p-phosphoinositide 3 kinase (PI3K; cat. no. ab182651) and PI3K (cat. no. ab40755) were obtained from Abcam (Shanghai, China). A polyclonal goat CY5-conjugated secondary antibody (cat. no. A10523) was from Thermo Fisher Scientific, Inc. (Waltham, MA, USA). An ROS assay kit and a Cell Counting kit-8 (CCK-8) were from the Beyotime Institute of Biotechnology (Haimen, China). Fetal bovine serum (FBS) and Dulbecco's modified Eagle's medium (DMEM) were provided by Gibco; Thermo Fisher Scientific, Inc. (Waltham, MA, USA).

Ethics and animal protocols. A total of 20 Sprague-Dawley rats (150-180 g, 6 weeks of age) were obtained from the Laboratory Animal Center of the Third Military Medical University (Chongqing, China). The animals had free access to food and water and were housed at a temperature of $25^{\circ} \mathrm{C}$ with $45-70 \%$ humidity, under a 12-h light/dark cycle in the specific pathogen free facility at Laboratory Animal Center of Third Military Medical University (Chongqing, China). All procedures were conducted in accordance with the animal care guidelines approved by the Animal Ethics Committee of the Third Military Medical University. The animal care and procedures were in accordance with the guidelines of the National Institutes of Health (Bethesda, MD, USA). Thoracic aortas were isolated from euthanized rats, and all efforts were made to minimize any pain to the animals.
Isolation and culture of VSMCs. Primary rat VSMCs were isolated from the thoracic aorta of Sprague-Dawley rats by an explant method as previously described (21). Briefly, male Sprague-Dawley rats were anaesthetized with 5\% chloral hydrate $(1 \mathrm{ml} / 100 \mathrm{~g}$ body weight $)$ and sacrificed to obtain VSMCs from the thoracic aorta. VSMCs were cultured in DMEM supplemented with $10 \% \mathrm{FBS}$ at $37^{\circ} \mathrm{C}$ in a humidified atmosphere containing $5 \% \mathrm{CO}_{2}$. The medium was changed every 2-3 days, and VSMCs at passages 3-8 were used for all experiments.

Analysis of cellular senescence. Senescent cells were identified using a Senescence $\beta$-Galactosidase Staining kit. Briefly, VSMCs were seeded into 6-well plates (NEST, Wuxi, China) at $2 \times 10^{5} /$ well. At $80 \%$ confluence, VSMCs were pretreated with celastrol at 10,50 or $100 \mathrm{nM}$ for $12 \mathrm{~h}$ before the addition of $100 \mathrm{nM}$ Ang II for 2 days. Subsequently, cells were fixed with the fixative solution, rinsed twice with PBS and incubated with the $\beta$-galactosidase staining solution at $37^{\circ} \mathrm{C}$ overnight in a dry incubator (without $\mathrm{CO}_{2}$ ). For $3 \mathrm{Ma}$ or Baf A1 stimulation, cells were pretreated with $3 \mathrm{mM} 3 \mathrm{Ma}$ or $50 \mathrm{nM}$ Baf A1 for $2 \mathrm{~h}$.

Cell cycle analysis. The cell cycle analysis was performed according to the manufacturer's protocol. Briefly, after serum starvation for $12 \mathrm{~h}$, VSMCs were treated with $50 \mathrm{nM}$ celastrol for $12 \mathrm{~h}$ before incubation with Ang II for 2 days at $37^{\circ} \mathrm{C}$. Subsequently, cells were harvested and fixed in $70 \%$ ethanol, stained for DNA content with propidium iodide for $30 \mathrm{~min}$ at room temperature and detected using a flow cytometer (BD FACScan, BD Biosciences, Franklin Lakes, NJ, USA). Fluorescence dot plots were analyzed and reconstructed with FlowJo version 7.2.5 software (FlowJo LLC, Ashland, OR, USA).

Western blot analysis. Western blot assays were performed as previously described (22). Briefly, VSMCs were harvested and lysed in RIPA buffer (Beyotime Institute of Biotechnology, Haimen, China), which contained a cocktail of both protease and phosphatase inhibitors (Roche Diagnostics, Basel, Switzerland). Following extraction from VSMCs, the protein concentration was determined by the BCA protein assay (Beyotime Institute of Biotechnology). Samples containing $30 \mu \mathrm{g}$ of protein were subjected to $10 \%$ SDS-PAGE and separated. Following protein transfer to nitrocellulose membranes (EMD Millipore), the membranes were blocked and incubated with primary antibodies overnight $(12 \mathrm{~h})$ at $4^{\circ} \mathrm{C}$, then washed three times with TBST (Tris-buffered saline; $10 \mathrm{mM}$ Tris- $\mathrm{HCl}$ $\mathrm{pH} 7.5,150 \mathrm{mM} \mathrm{NaCl}$ and $0.1 \%$ Tween-20) for $30 \mathrm{~min}$ and incubated with secondary antibodies for $1 \mathrm{~h}$ at room temperature. Membranes were washed four times with TBST for $40 \mathrm{~min}$. The protein signals were detected using the SuperSignal West Pico Chemiluminescent Substrate (Pierce; Thermo Fisher Scientific, Inc.). The relative protein levels were analyzed by Image J software version 1.6.0_24 (National Institutes of Health, Bethesda, MD, USA).

Determination of intracellular ROS. A ROS assay kit was used to determine the intracellular ROS generation. In brief, quiescent cells were incubated with $50 \mathrm{nM}$ celastrol for $12 \mathrm{~h}$ before stimulation with $100 \mathrm{nM}$ Ang II for $12 \mathrm{~h}$, and then 
incubated with 2, 7-DCFH-DA in DMEM without FBS at $37^{\circ} \mathrm{C}$ for $30 \mathrm{~min}$ and rinsed three times with PBS. ROS levels were measured by using a flow cytometer.

Immunofluorescent staining of LC3. Cells were fixed with $4 \%$ paraformaldehyde for $20 \mathrm{~min}$, rinsed three times with PBS, blocked with $1 \%$ bovine serum albumin (Beyotime Institute of Biotechnology) for $1 \mathrm{~h}$, and incubated with a rabbit anti-LC3 A/B antibody for $1 \mathrm{~h}$ at room temperature. Subsequently, the cells were washed and incubated with a CY5-conjugated secondary antibody for $1 \mathrm{~h}$ at room temperature. Immunofluorescence was detected by using a confocal microscope (TCS-SP5, Leica Microsystems GmbH, Wetzlar, Germany).

Statistical analysis. Data analysis was performed using GraphPad Prism 5.0 software package (GraphPad Software, Inc., La Jolla, CA, USA). All experimental data are expressed as the mean \pm standard error with each experiment repeated at least three times. The statistical analysis was performed with SPSS software version 20.0 (IBM Corp., Armonk, NY, USA). The data comparisons were analyzed using one-way analysis of variance followed by Bonferroni's multiple comparisons test. $\mathrm{P}<0.05$ was considered to indicate a statistically significant difference.

\section{Results}

Celastrol treatment counteracts Ang II-induced cellular senescence in VSMCs. Senescent cells are characterized by stable growth arrest (2) and increased expression of SA- $\beta$-gal, p53, p21 and p16 $(3,4)$. Treatment with celastrol alone had little effect on VSMC senescence compared with treatment with the vehicle control (DMSO). A significant increase in VSMC senescence upon stimulation with Ang II was evidenced by the increase in the number of SA- $\beta$-gal-positive cells. In this setting, celastrol demonstrated a dose-dependent inhibition of VSMC senescence with its maximal effect achieved at $50 \mathrm{nM}$ (Fig. 1A). Protein expression levels of p53 and p21 were significantly increased in VSMCs after a 2-day period of stimulation with Ang II. Pretreatment with celastrol significantly counteracted this effect of Ang II on (Fig. 1B). Flow cytometry was also used to investigate the effect of celastrol on the cell cycle of VSMCs. The senescent cells were defined as arrested primarily in the G0/G1 phase but occasionally at the S or G2 phase. After stimulation with $100 \mathrm{nM}$ Ang II for 2 days, the percentage of G0/G1 phase cells was significantly increased compared with that of the DMSO control group. In contrast, in VSMCs treated with Ang II, the number of G0/G1-arrested cells was decreased while the number of cells in S/G2 phase was increased by celastrol (50 nM)-pretreatment (Fig. 1C), indicating that the proliferative capacity of senescent VSMCs induced by Ang II was restored by celastrol.

Celastrol alleviates senescence through reducing ROS activity in VSMCs. Intracellular ROS serves a major role in the development of Ang II-induced cellular senescence (23), which can be alleviated by the specific ROS scavenger NAC in VSMCs (8). A ROS assay kit was used to investigate whether celastrol attenuated the Ang II-induced ROS activity in VSMCs. Treatment with celastrol alone had little effect on basal ROS generation, similar to that of the DMSO control (Fig. 1D). ROS production was increased in the Ang II-treated group and this was significantly decreased in the celastrol-pretreated group, although such inhibitory efficacy was less potent compared with cells treated with NAC (Fig. 1D). These results suggested that the effects of celastrol on cellular senescence in VSMCs are potentially mediated through the inhibition of ROS production.

Celastrol-induced autophagy reduces Ang II-mediated senescence in VSMCs. Immunofluorescence assay demonstrated that the level of total LC3 was significantly increased in the celastrol-pretreated group compared with the control group (Fig. 2A). Western blot assay further demonstrated that compared with the DMSO control, the protein expression level of LC3 II was increased in the celastrol-treated group, which could be further increased by bafilomycin A1, or decreased by $3 \mathrm{Ma}$ (Fig. 2B). Furthermore, the p62 protein level was decreased in the celastrol-treated group, and treatment with the two autophagy inhibitors reduced these effects (Fig. 2B). Ang II stimulation significantly increased cellular ROS levels in VSMCs, and celastrol reduced the Ang II-stimulated ROS levels, similar to the effects of the positive control rapamycin; however, pretreating with the autophagy inhibitor $3 \mathrm{Ma}$ reversed the effect of celastrol (Fig. 2C), suggesting that celastrol at least partially inhibits intracellular ROS by activating autophagy in VSMCs.

To further confirm whether celastrol-induced autophagy can reduce the Ang II-induced senescence of VSMCs, senescence $\beta$-galactosidase staining and western blot analysis were performed. The results demonstrated that, just like in the autophagy inducer rapamycin-pretreated group, the SA- $\beta$-gal positive cell counts were significantly decreased in the celastrol-pretreated group, which was reversed by the autophagy inhibitors 3Ma and Baf A1 (Fig. 3A). Western blot assay further demonstrated that the autophagy inhibitor 3Ma largely abolished the effects of celastrol on p53 and p21 expression (Fig. 3B).

Celastrol inhibits the PI3K/Akt/mTOR signaling pathway. The present study then assessed tested whether celastrol could suppress the PI3K/Akt/mTOR signaling pathway. The results demonstrated that, compared with the control group, the phosphorylation levels of PI3K, Akt, mTOR and p70S6K in the celastrol group were downregulated (Fig. 4), indicating that celastrol treatment suppressed the PI3K/Akt/mTOR signaling pathway.

\section{Discussion}

It has been reported that treatment with tripterine (celastrol) decreases ox-LDL-induced oxidative stress, apoptosis and senescence of endothelial progenitor cells (16). However, whether celastrol exerts any effects on VSMC senescence remains unknown. The present study provided, to the best of our knowledge, the first evidence that celastrol upregulates autophagy which reduces ROS levels, and subsequently counteracts Ang II-induced cellular senescence. Meanwhile, celastrol inhibited the PI3K/Akt/mTOR signaling pathway 


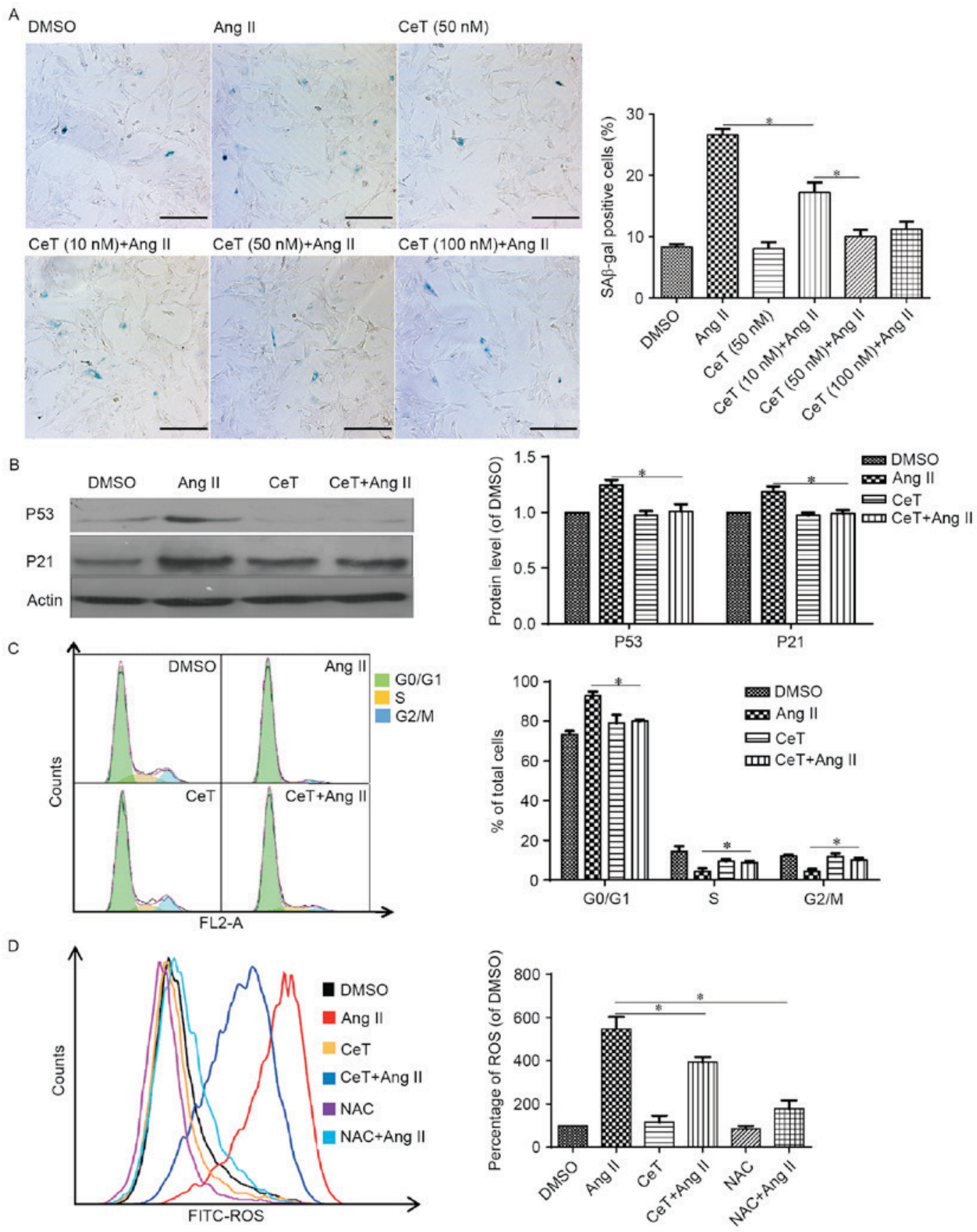

Figure 1. Celastrol treatment reduces Ang II-induced senescence by reducing ROS activity in VSMCs. (A) Senescence-associated $\beta$-galactosidase staining of VSMCs. The blue puncta indicate the senescent cells. Scale bar $=250 \mu \mathrm{m}$. (B) Western blot measuring the effects of celastrol treatment on the protein expression of p53 and p21 induced by Ang II in VSMCs. (C) Cell cycle analysis using flow cytometry. The bars show the percentage of cells in the different phases (G1/G0, S, and G2/M phase) compared with control. (D) Flow cytometric analysis of ROS in celastrol-treated cells. The representative flow cytometry results and statistical analysis are presented. Data are presented as the mean \pm standard error. ${ }^{*} \mathrm{P}<0.05(\mathrm{n}=3)$. CeT, celastrol. Ang II, angiotensin II; NAC, $N$-acetyl-L-cysteine; ROS, reactive oxygen species; FITC, fluorescein isothiocyanate. VSMCs, vascular smooth muscle cells. SA $\beta$-gal, senescence-associated $\beta$-galactosidase; SA $\beta$-gal, senescence-associated $\beta$-galactosidase; VSMCs, vascular smooth muscle cells; DMSO, dimethyl sulfoxide.

in VSMCs. Senescence contributes to the development of aging-associated pathologies such as cardiovascular disorders, diabetes and neurodegenerative diseases $(20,24)$.

ROS is an important senescence-inducing stressor for vascular cells and can induce certain DNA lesions and drive senescence, either dependent or independent of telomere shortening (25). ROS has been implicated in Ang II-induced cellular senescence (7) and interventions to decrease ROS production by both scavenging free radicals and enhancing antioxidant defense have been widely proposed as an anti-aging strategy (24). To the best of our knowledge, the present study revealed for the first time that pretreatment with celastrol reduces the level of Ang II-induced ROS.

Autophagy has attracted growing interest because it affects almost every organ system and modulates an expanding list of disease processes (18). Autophagy contributes to general homeostasis through the turnover of long-lived proteins and organelles via lysosomal degradation. Recent studies have suggested that autophagy is relevant to human diseases $(20,26)$. Researchers have investigated the association 
A
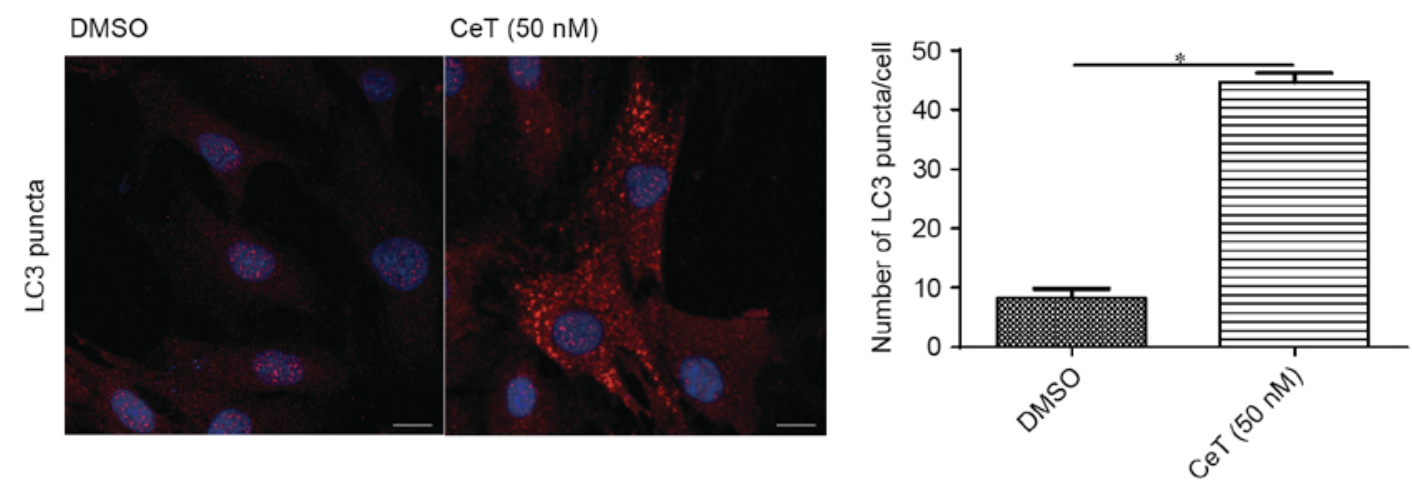

B
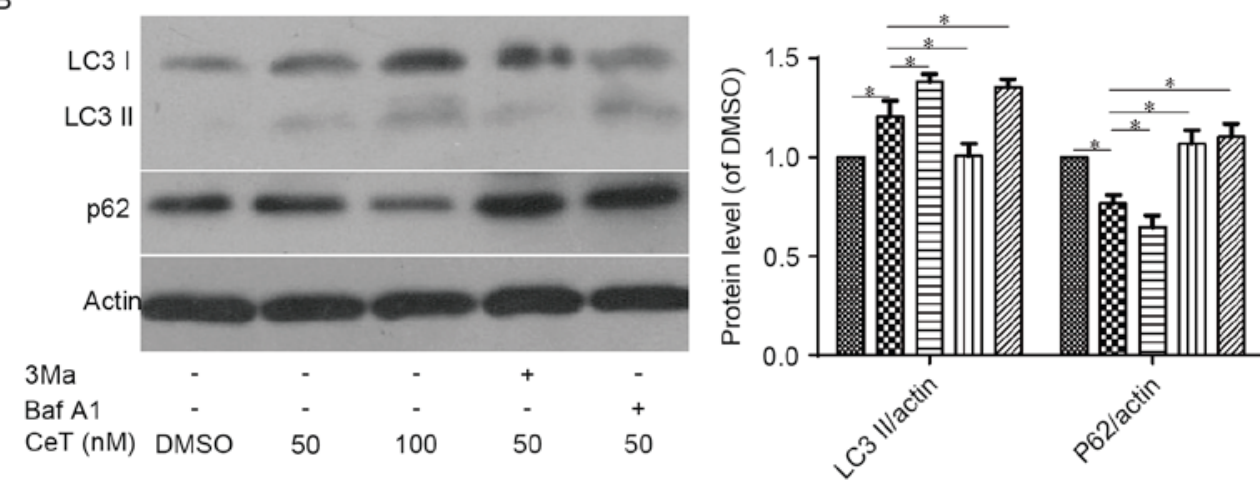

DMSO

$\mathbf{C e T}(50 \mathrm{nM})$

$\square$ CeT $(100 \mathrm{nM})$

m $\mathrm{CeT}(50 \mathrm{nM})+3 \mathrm{Ma}$

m CeT $(50 \mathrm{nM})+\mathrm{Baf} A 1$

C
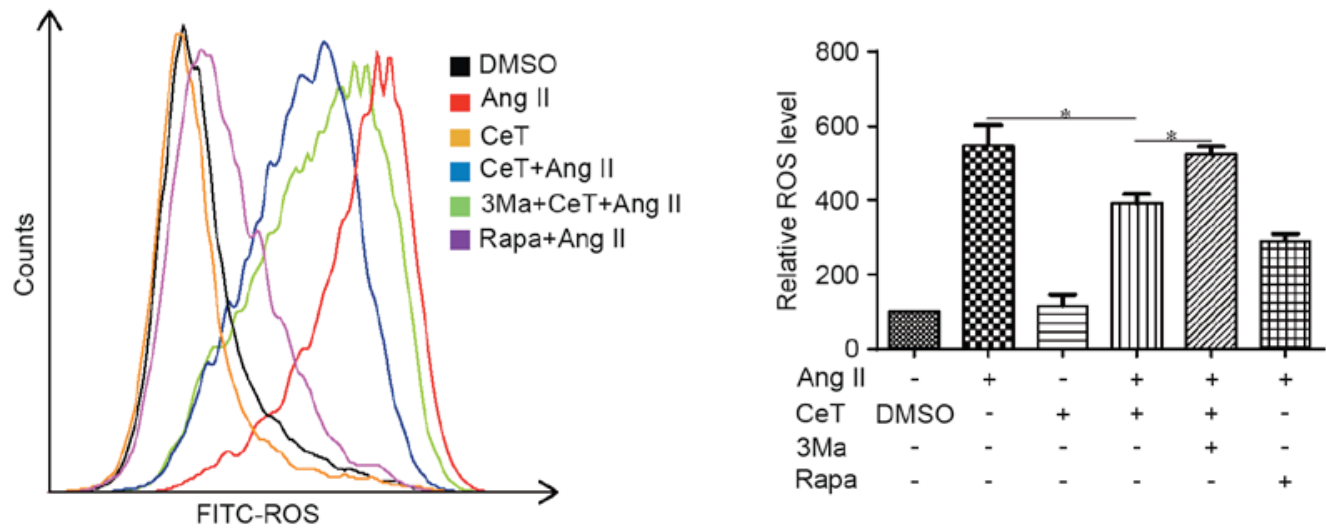

Figure 2. Celastrol induces autophagy in VSMCs. Serum-starved VSMCs were pre-incubated with varying concentrations of either celastrol or vehicle $(0.1 \%$ DMSO) for $12 \mathrm{~h}$ and then (A) examined using immunofluorescence assay to identify LC3 puncta or (B) subjected to western blotting to determine the protein levels of LC3 and p62. Scale bar=10 $\mu \mathrm{m}$. (C) Flow cytometric analysis of ROS in celastrol-treated cells. Data are presented as the mean \pm standard error. ${ }^{*} \mathrm{P}<0.05(\mathrm{n}=3)$. CeT, celastrol; Ang II, angiotensin II. LC3, microtubule-associated protein light chain 3; 3Ma, 3-methyladenine. Baf A1, bafilomycin A1; Rapa, rapamycin; FITC, fluorescein isothiocyanate; ROS, reactive oxygen species; VSMCs, vascular smooth muscle cells; DMSO, dimethyl sulfoxide.

between autophagy and aging over the years. The induction of autophagy by caloric restriction, spermidine, resveratrol or rapamycin can extend the life span of different organisms (27). The autophagy inducers rapamycin and resveratrol suppress cellular senescence by partially preventing the loss of proliferative potential $(28,29)$, whereas defective autophagy in VSMCs accelerates senescence and promotes ligation-induced neointima formation and diet-induced atherogenesis (20). It has been demonstrated that autophagy reduces ROS (17). By upregulation of autophagy, celastrol protects the SH-SY5Y human neuroblastoma cell line from rotenone-induced injury (30) and ameliorates experimental colitis in interleukin-10-deficient mice (31). However, whether celastrol can induce autophagy and subsequently exert a protective effect on VSMCs is still unknown. In the present study, novel evidence is presented for a prominent role of celastrol-activated autophagy in regulating ROS to reduce VSMC senescence.

In addition, celastrol has been reported to affect a diverse range of cellular functions that involves various signaling pathways. For example, celastrol protects against obesity and metabolic dysfunction through activation of a HSF1-PGCla transcriptional axis (11). Celastrol attenuates hypertension-induced inflammation and oxidative stress in VSMCs via induction of heme oxygenase-1 (32). However, there have been no reports about the direct target or binding receptor of celastrol in a cell yet, which makes it difficult to explain the mechanisms for what the reported studies observed. It is well known that the PI3K/Akt/mTOR pathway mediates an inhibitory signal on autophagy (33). Several studies have implicated that celastrol induces autophagy via the suppression of the 
A
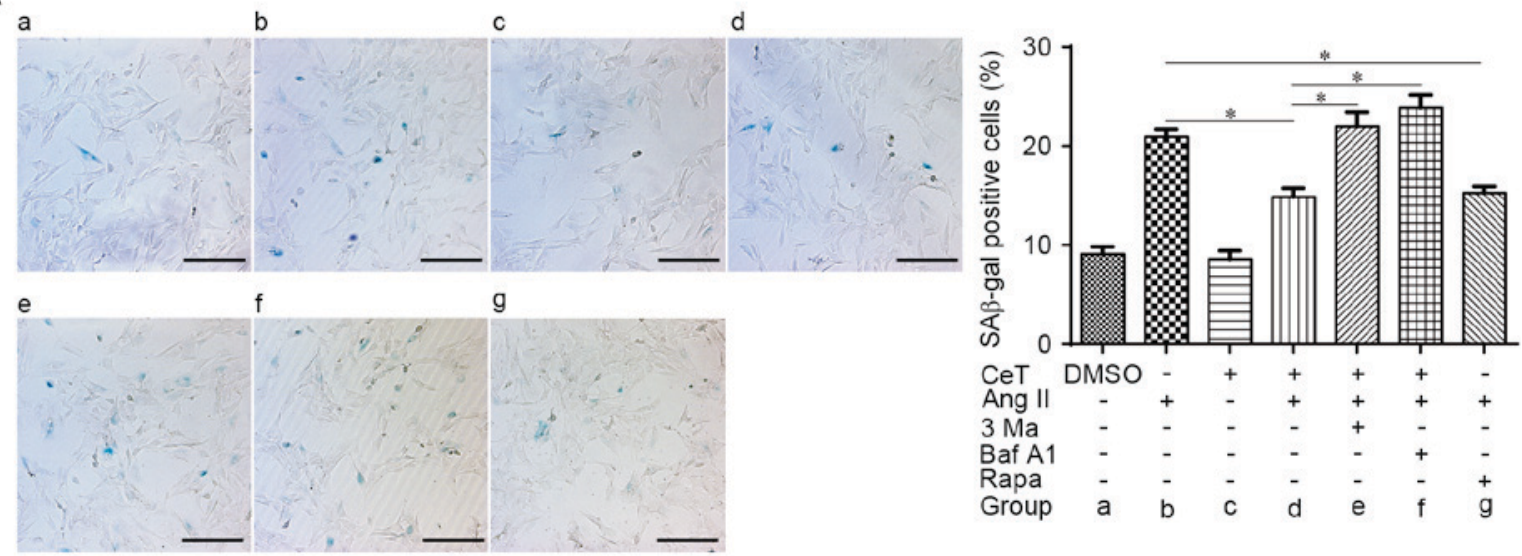

B
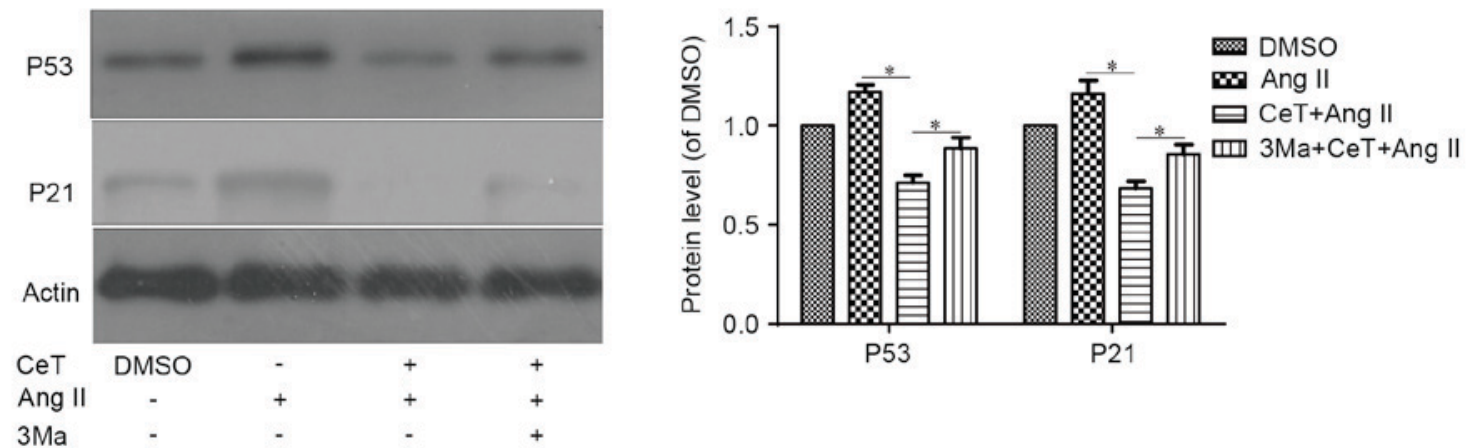

Figure 3. Autophagy inhibitors reverse the effects of celastrol on VSMC senescence. (A) SA $\beta$-gal staining of VSMCs. Scale bar=250 $\mu$ m. (B) Western blot measuring the effects of 3Ma treatment on the protein expression levels of p53 and p21 in VSMCs. Data are presented as the mean \pm standard error. ${ }^{*} \mathrm{P}<0.05$ ( $\mathrm{n}=3$ ). CeT, celastrol; SA $\beta$-gal, senescence-associated $\beta$-galactosidase; Ang II, angiotensin II; 3Ma, 3-methyladenine; Baf A1, bafilomycin A1; Rapa, Rapamycin; VSMCs, vascular smooth muscle cells; DMSO, dimethyl sulfoxide.

PI3K/Akt/mTOR pathway in the proximal colon of mice and in tumor cells $(31,34)$. The present study demonstrated that celastrol significantly suppressed the PI3K/Akt/mTOR signaling pathway and upregulated autophagy; celastrol reduces ROS by inducing autophagy in VSMCs by using an autophagy inhibitor. Thus, though the direct target or receptor of celastrol in VSMCs remains unknown, the present study revealed the close association between celastrol and the PI3K/Akt/mTOR-autophagy-ROS axis, i.e. that celastrol possibly promotes autophagy via the PI3K/Akt/mTOR pathway in VSMCs. However, it cannot be concluded that the effect of autophagy upregulation by celastrol is precisely $\mathrm{PI} 3 \mathrm{~K} / \mathrm{Akt} / \mathrm{mTOR}$ dependent, because specific inhibitors or specific small interfering RNAs of PI3K/Akt/mTOR were not used in this study. Therefore, there may be other possible mechanisms through which celastrol may function, as opposed to a direct effect on PI3K/Akt/mTOR. Nevertheless, this issue could be addressed in the future by investigating the effects of celastrol on autophagy with or without administration of the specific inhibitors or siRNAs of PI3K/Akt/mTOR axis in the same cell model.

As one of the biomarkers of autophagy used in these experiments, LC3 protein is involved in phagophore formation, which is the primary autophagy-related protein (Atg) 8 homolog examined in mammalian cells (35), and p62 is constantly degraded via autophagy through its LIR domain that binds to LC3 on the membranes of autophagosomes (36).
The concomitant increase in LC3-II and degradation of p62 typically reflect the potent induction of autophagy (35). The present study observed increased LC3-II and decreased p62 levels in VSMCs by celastrol treatment, which may be due to the inhibition of PI3K/Akt/mTOR signaling pathway by celastrol, as mTOR is a classic negative regulator of autophagy (33). In addition, though there are several autophagy markers such as LC3, Atg5, Atg7, Atg9, Atg12-Atg5, ATG14, ATG16L1 and p62 (35), only LC3 and p62 were selected as representative markers, as investigated previously $(37,38)$ to assess the induction of autophagy by celastrol, thus; whether other autophagy markers are also affected by celastrol has to be further verified in future work.

The present study demonstrated that celastrol upregulated autophagy, which reduced ROS and subsequently counteracted the cellular senescence of VSMCs as a consequence of Ang II stimulation. However, it has also been revealed that celastrol suppresses the viability of cancer cells through AMPK activation, which is also caused by ROS generation (39). In this context, celastrol was demonstrated to induce prolonged and excessive autophagy in tumor cells, leading to cell death (31). In these studies, celastrol was used at a much higher dose compared with the dose $(50 \mathrm{nM})$ tested in the present study, which is consistent with some reports, in which either a moderate dose or short duration treatment of celastrol induced an anti-inflammatory or anti-autoimmune effect $(10,40)$. To avoid inducing intracellular ROS and excessive autophagy, 


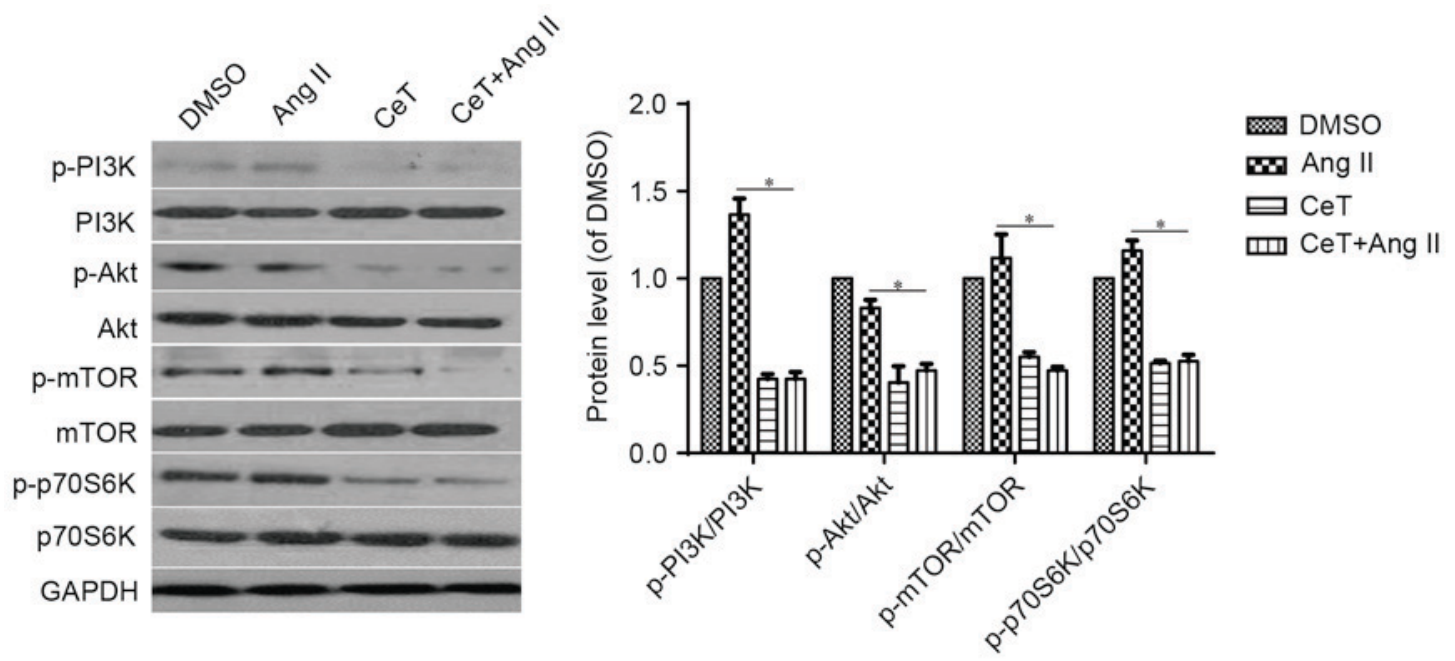

Figure 4. Celastrol suppresses the PI3K/Akt/mTOR signaling pathway. Representative western blot images and quantification of protein expression levels of $\mathrm{p}$-PI3K, p-Akt, p-mTOR and p-p70S6K in VSMCs. GAPDH served as an internal control. Data are presented as the mean \pm standard error. "P<0.05 (n=3). CeT, celastrol; Ang II, angiotensin II; VSMCs, vascular smooth muscle cells; p, phosphorylated; Akt, protein kinase B; mTOR, mechanistic target of rapamycin; PI3K, phosphoinositide 3 kinase; DMSO, dimethyl sulfoxide; p70s6k, ribosomal protein S6 kinase $\beta 1$.

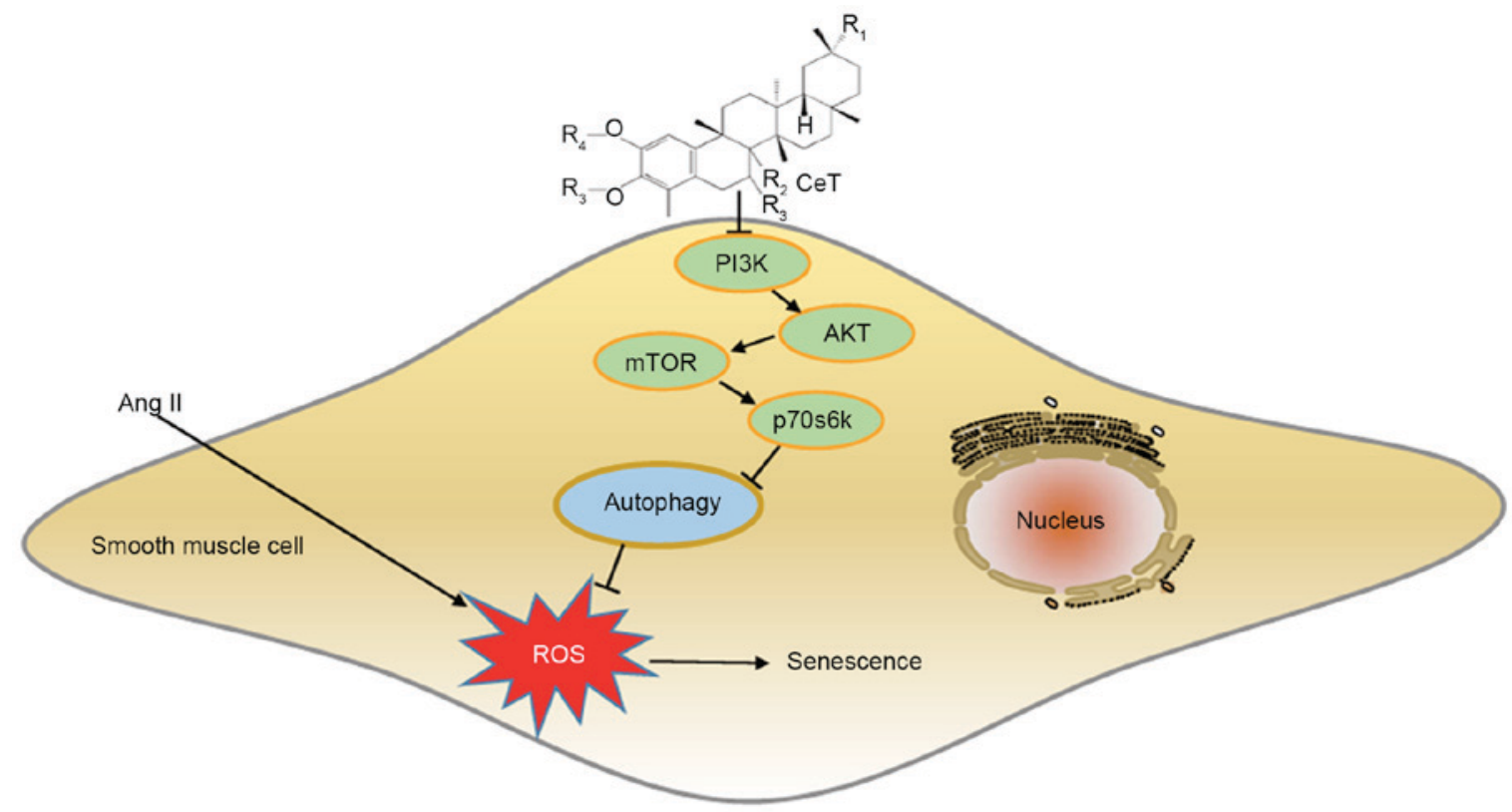

Figure 5. Schematic diagram of the effect of CeT on Ang II-induced cellular senescence in VSMCs. Ang II enhances production of reactive oxygen species that ultimately lead to cellular senescence. Celastrol upregulates autophagy that may be associated with the PI3K/Akt/ mTOR signaling pathway, which further inhibits ROS production and attenuates VSMC senescence induced by Ang II. CeT, celastrol; Ang II, angiotensin II; VSMCs, vascular smooth muscle cells; Akt, protein kinase B; mTOR, mechanistic target of rapamycin; PI3K, phosphoinositide 3 kinase; p70s6k, ribosomal protein S6 kinase $\beta 1$.

which can lead to apoptosis and cell death, the present study conducted a cytotoxic experiment to optimize the celastrol dose to a moderate level. The results demonstrated that celastrol did not influence the viability of VSMCs at a dose up to $50 \mathrm{nM}$ (data not shown). In general, these results demonstrated that treatment with a moderate dose of celastrol $(50 \mathrm{nM})$ on VSMCs in vitro can significantly exert a positive effect on VSMCs.

In conclusion, the present study demonstrated that celastrol upregulates autophagy, which confers resistance to Ang II-induced superoxide generation and the resultant senescence. Notably, the effects of celastrol on stimulating autophagy may be associated with its inhibition of mTOR activity (Fig. 5). As VSMC senescence is present in atherosclerotic plaques and contributes to the pathogenesis of atherosclerosis, celastrol may hold promise for the prevention and treatment of cardiovascular diseases.

\section{Acknowledgements}

The present study was supported by the grants from the National Natural Science Foundation of China (grant nos. 81270362 and 81470561) and the State Project for Essential Drug Research and Development (grant 
no. 2013ZX09103003-001). The manuscript language has been corrected by American Journal Experts, (certificate verification key: 9290-E20B-063D-B6AD-8954).

\section{References}

1. Wang JC and Bennett M: Aging and atherosclerosis: Mechanisms, functional consequences, and potential therapeutics for cellular senescence. Circ Res 111: 245-259, 2012.

2. Childs BG, Durik M, Baker DJ and van Deursen JM: Cellular senescence in aging and age-related disease: From mechanisms to therapy. Nat Med 21: 1424-1435, 2015.

3. Dimri GP, Lee X, Basile G, Acosta M, Scott G, Roskelley C, Medrano EE, Linskens M, Rubelj I, Pereira-Smith O, et al: A biomarker that identifies senescent human cells in culture and in aging skin in vivo. Proc Natl Acad Sci USA 92: 9363-9367, 1995.

4. Kunieda T, Minamino T, Nishi J, Tateno K, Oyama T, Katsuno T, Miyauchi H, Orimo M, Okada S, Takamura M, et al: Angiotensin II induces premature senescence of vascular smooth muscle cells and accelerates the development of atherosclerosis via a p21-dependent pathway. Circulation 114: 953-960, 2006.

5. Wiley CD, Velarde MC, Lecot P, Liu S, Sarnoski EA, Freund A, Shirakawa K, Lim HW, Davis SS, Ramanathan A, et al: Mitochondrial dysfunction induces senescence with a distinct secretory phenotype. Cell Metab 23: 303-314, 2016.

6. Correia-Melo C, Hewitt G and Passos JF: Telomeres, oxidative stress and inflammatory factors: Partners in cellular senescence? Longev Healthspan 3: 1, 2014.

7. Herbert KE, Mistry Y, Hastings R, Poolman T, Niklason L and Williams B: Angiotensin II-mediated oxidative DNA damage accelerates cellular senescence in cultured human vascular smooth muscle cells via telomere-dependent and independent pathways. Circ Res 102: 201-208, 2008.

8. Zhao L, Li AQ, Zhou TF, Zhang MQ and Qin XM: Exendin-4 alleviates angiotensin II-induced senescence in vascular smooth muscle cells by inhibiting Racl activation via a cAMP/PKA-dependent pathway. Am J Physiol Cell Physiol 307: C1130-C1141, 2014.

9. Lee KY, Kim JR and Choi HC: Genistein-induced LKB1-AMPK activation inhibits senescence of VSMC through autophagy induction. Vascul Pharmacol 81: 75-82, 2016.

10. Bian M, Du X, Cui J, Wang P, Wang W, Zhu W, Zhang T and Chen Y: Celastrol protects mouse retinas from bright light-induced degeneration through inhibition of oxidative stress and inflammation. J Neuroinflammation 13: 50, 2016.

11. Ma X, Xu L, Alberobello AT, Gavrilova O, Bagattin A, Skarulis M, Liu J, Finkel T and Mueller E: Celastrol protects against obesity and metabolic dysfunction through activation of a HSF1-PGC1 $\alpha$ transcriptional axis. Cell Metab 22: 695-708, 2015.

12. Wang YL, Lam KK, Cheng PY and Lee YM: Celastrol prevents circulatory failure via induction of heme oxygenase-1 and heat shock protein 70 in endotoxemic rats. J Ethnopharmacol 162: $168-175,2015$

13. Gu L, Bai W, Li S, Zhang Y, Han Y, Gu Y, Meng G, Xie L, Wang J, Xiao Y, et al: Celastrol prevents atherosclerosis via inhibiting LOX-1 and oxidative stress. PLoS One 8: e65477, 2013.

14. Der Sarkissian S, Cailhier JF, Borie M, Stevens LM, Gaboury L, Mansour S, Hamet P and Noiseux N: Celastrol protects ischaemic myocardium through a heat shock response with up-regulation of haeme oxygenase-1. Br J Pharmacol 171: 5265-5279, 2014.

15. Hu H, Straub A, Tian Z, Bassler N, Cheng J and Peter K: Celastrol, a triterpene extracted from Tripterygium wilfordii Hook F, inhibits platelet activation. J Cardiovasc Pharmacol 54: 240-245, 2009

16. Lu C, Zhang X, Zhang D, Pei E, Xu J, Tang T, Ye M, Uzan G, Zhi K, Li M and Zuo K: Short time tripterine treatment enhances endothelial progenitor cell function via heat shock protein 32 J Cell Physiol 230: 1139-1147, 2015.

17. Li L, Tan J, Miao Y, Lei P and Zhang Q: ROS and Autophagy: Interactions and molecular regulatory mechanisms. Cell Mol Neurobiol 35: 615-621, 2015.

18. Tai S, Hu XQ, Peng DQ, Zhou SH and Zheng XL: The roles of autophagy in vascular smooth muscle cells. Int J Cardiol 211: 1-6, 2016.
19. Nussenzweig SC, Verma S and Finkel T: The role of autophagy in vascular biology. Circ Res 116: 480-488, 2015.

20. Grootaert MO, da Costa Martins PA, Bitsch N, Pintelon I, De Meyer GR, Martinet W and Schrijvers DM: Defective autophagy in vascular smooth muscle cells accelerates senescence and promotes neointima formation and atherogenesis. Autophagy 11: 2014-2032, 2015.

21. Feng S, Hu Y, Peng S, Han S, Tao H, Zhang Q, Xu X, Zhang J and $\mathrm{Hu} \mathrm{H}$ : Nanoparticles responsive to the inflammatory microenvironment for targeted treatment of arterial restenosis. Biomaterials 105: 167-184, 2016.

22. Chen Q, Bei JJ, Liu C, Feng SB, Zhao WB, Zhou Z, Yu ZP, Du XJ and Hu HY: HMGB1 induces secretion of matrix vesicles by macrophages to enhance ectopic mineralization. PLoS One 11: e0156686, 2016.

23. Tsai IC, Pan ZC, Cheng HP, Liu CH, Lin BT and Jiang MJ: Reactive oxygen species derived from NADPH oxidase 1 and mitochondria mediate angiotensin II-induced smooth muscle cell senescence. J Mol Cell Cardiol 98: 18-27, 2016.

24. Davalli P, Mitic T, Caporali A, Lauriola A and D'Arca D: ROS cell senescence, and novel molecular mechanisms in aging and age-related diseases. Oxid Med Cell Longev 2016: 3565127, 2016.

25. Yin $\mathrm{H}$ and Pickering JG: Cellular senescence and vascular disease: Novel routes to better understanding and therapy. Can J Cardiol 32: 612-623, 2016.

26. Zhong Z, Sanchez-Lopez E and Karin M: Autophagy, inflammation, and immunity: A troika governing cancer and its treatment. Cell 166: 288-298, 2016.

27. Rubinsztein DC, Marino G and Kroemer G: Autophagy and aging. Cell 146: 682-695, 2011

28. Shan H, Guo D, Li X, Zhao X, Li W and Bai X: From autophagy to senescence and apoptosis in Angiotensin II-treated vascular endothelial cells. APMIS 122: 985-992, 2014.

29. Mei Y, Thompson MD, Cohen RA and Tong X: Autophagy and oxidative stress in cardiovascular diseases. Biochim Biophys Acta 1852: 243-251, 2015.

30. Deng YN, Shi J, Liu J and Qu QM: Celastrol protects human neuroblastoma SH-SY5Y cells from rotenone-induced injury through induction of autophagy. Neurochem Int 63: 1-9, 2013.

31. Zhao J, Sun Y, Shi P, Dong JN, Zuo LG, Wang HG, Gong JF, Li Y, Gu LL, Li N, et al: Celastrol ameliorates experimental colitis in IL-10 deficient mice via the up-regulation of autophagy. Int Immunopharmacol 26: 221-228, 2015.

32. Yu X, Tao W, Jiang F, Li C, Lin J and Liu C: Celastrol attenuates hypertension-induced inflammation and oxidative stress in vascular smooth muscle cells via induction of heme oxygenase-1. Am J Hypertens 23: 895-903, 2010.

33. Shinojima N, Yokoyama T, Kondo Y and Kondo S: Roles of the Akt/mTOR/p70S6K and ERK1/2 signaling pathways in curcumin-induced autophagy. Autophagy 3: 635-637, 2007.

34. Lee HW, Jang KS, Choi HJ, Jo A, Cheong JH and Chun KH: Celastrol inhibits gastric cancer growth by induction of apoptosis and autophagy. BMB Rep 47: 697-702, 2014.

35. Klionsky DJ, Abdelmohsen K, Abe A, Abedin MJ, Abeliovich $\mathrm{H}$, Acevedo Arozena A, Adachi H, Adams CM, Adams PD, Adeli K, et al: Guidelines for the use and interpretation of assays for monitoring autophagy (3rd edition). Autophagy 12: 1-222, 2016.

36. Moscat J and Diaz-Meco MT: p62 at the crossroads of autophagy, apoptosis, and cancer. Cell 137: 1001-1004, 2009.

37. Rodriguez-Arribas M, Yakhine-Diop SM, González-Polo RA, Niso-Santano M and Fuentes JM: Turnover of lipidated LC3 and autophagic cargoes in mammalian cells. Methods Enzymol 587: $55-70,2017$

38. Wang S, Wang C, Yan F, Wang T, He Y, Li H, Xia Z and Zhang Z: $\mathrm{N}$-acetylcysteine attenuates diabetic myocardial ischemia reperfusion injury through inhibiting excessive autophagy. Mediators Inflamm 2017: 9257291, 2017.

39. Kim JH, Lee JO, Lee SK, Kim N, You GY, Moon JW, Sha J, Kim SJ, Park SH and Kim HS: Celastrol suppresses breast cancer MCF-7 cell viability via the AMP-activated protein kinase (AMPK)-induced p53-polo like kinase 2 (PLK-2) pathway. Cell Signal 25: 805-813,2013.

40. Yu Y, Koehn CD, Yue Y, Li S, Thiele GM, Hearth-Holmes MP, Mikuls TR, O'Dell JR, Klassen LW, Zhang Z and Su K: Celastrol inhibits inflammatory stimuli-induced neutrophil extracellular trap formation. Curr Mol Med 15: 401-410, 2015. 